\title{
Le travail d'une Commission nationale d'éthique
}

\section{Jean Martin}

Dr méd., membre de la rédaction

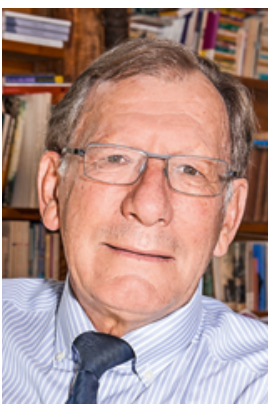

Au numéro 3/2017 du Hastings Center Report est joint un Rapport spécial traitant des Commissions nationales d'éthique. On se souvient que le pionnier en la matière est le Comité consultatif national français (CCNE), créé en 1983, et que la Commission nationale suisse (CNE) a été instituée en 2001. ${ }^{1}$ Membre de cette dernière de 2001 à 2013, j’ai été intéressé par ce document, notamment le texte de l'éthicien connu Alexander Capron, de l'University of Southern California [1], qui discute le statut et les activités des sept commissions officielles qu'ont connues les USA (nommées par le Congrès ou le Président) depuis 1974.

\section{Membres, durée des mandats}

Dès la première, ses membres étaient issus d'une diversité de domaines. De plus, ils n'étaient/ne sont pas supposés être des représentants, des avocats corporatistes, du milieu dont ils émanent. Pour Capron, il importe que cela reste la règle. Il est judicieux aussi que, comme jusqu'ici, les serviteurs publics (de l'administration fédérale) ne puissent être membres. La commission doit être un groupe varié de personnalités réfléchies capables de délibérer constructivement et d'arriver à des conclusions d'une façon qui répond aux besoins de la société. A son avis, son mandat ne devrait pas être limité dans le temps (la continuité est une condition du succès), mais bien celui de ses membres.

\section{Fonctionnement, transparence, rapports}

Il importe que des organismes chargés de conseiller l'autorité fassent de l'éthique en public, dit Capron, condition salutaire parce que le fait de parler «on the record" (avec un enregistrement accessible) incite à être bien attentif à l'exactitude et la pertinence de ce qu'on affirme. Rendre publics ces travaux est bien entendu beaucoup facilité par Internet, qui fournit aussi la possibilité de feedback par la collectivité. Note de J.M.: j'ai parfois été ambivalent quant à cette exigence de "publicité». Oui, on est alors plus prudent dans ce qu'on dit mais cela peut enlever un certain caractère vif, voire provocant, aux débats.

Pratiquement, les Commissions américaines ont fonctionné de manière inductive, étudiant les questions dans leur contexte spécifique. Ceci plutôt que de vouloir articuler a priori un cadre de grands principes généraux. A une exception près, les travaux de ces commissions ont montré une forte tendance à rechercher le consensus. Capron: «Le seul vrai pouvoir d'une commission - le pouvoir de persuasion - est plus fort s'il y a unanimité.»

«Les rapports de commission doivent être intellectuellement respectables», dit-il, "mais leur audience prioritaire n'est pas constituée par des philosophes et autres experts académiques», mais bien par les élus à différents niveaux, la société civile et le public en général. Tout à fait d'accord. En général, on peut dire que cette évaluation d'outre-Atlantique (par une personnalité libérale, pas un membre de l'administration Trump) est proche de ce qui vaut pour notre CNE.

Une commission nationale doit bénéficier d'une totale indépendance dans ses travaux (et, en principe, quant au choix des sujets qu'elle étudie). Aux USA, elle a en plus la faculté (action-forcing authority) d'émettre des recommandations à l'attention d'une agence fédérale, lui enjoignant de prendre certaines mesures - élaborer rapidement des dispositions sur le sujet considéré et les mettre en consultation publique. Toutefois, en pratique, il s'est avéré difficile aux commissions de forcer un département gouvernemental à agir. ${ }^{2}$

Conclusion? «La médecine et la recherche n'ont pas fini de produire des dilemmes éthiques» (un vrai euphémisme); aujourd'hui, «le soleil ne se couche jamais sur le monde de la bioéthique». Et, alors que le président Trump ne semble pas pressé d'en nommer une, Capron dit l'importance de disposer d'un tel organe, auquel puissent être soumis sans délai les problèmes qui surgissent. La longévité du CCNE français et sa capacité à s'élever au-dessus du politique illustrent à son avis la valeur d'une instance permanente, plutôt que de commissions successives ne durant que le temps d'une législature.

\section{Références}

1 Capron A.M. Building the Next Bioethics Commission. In: Goals and Practice of Public Bioethics: Reflections on National Bioethics Commissions. Special Report, Hastings Center Report 47, Supplement to no. 3, May-June 2017, pages S4-S9. 